\title{
Efectividad para dejar de fumar de los cigarrillos electrónicos
}

\author{
Effectiveness of electronic cigarettes to stop smoking
}

\begin{abstract}
Alejandro Videla*, Mariano Bertoncini ${ }^{\dagger}$, Brunilda Casetta ${ }^{\ddagger}$, Paola Morello§,
\end{abstract} Bettina Forno ${ }^{\star \star}$, Natalie Gospodinoff ${ }^{\dagger}$, Ignacio Drake ${ }^{\ddagger \ddagger}$.

Videla A, Bertoncini M, Casetta B, Morello P, Forno B, Gospodinoff N, Drake I. Efectividad para dejar de fumar por parte de los cigarrillos electrónicos. Evid Actual Pract Ambul 2019;21(4):100-101.

\begin{abstract}
A partir de la aparición de diferentes notas periodísticas que destacan los potenciales beneficios que el cigarrillo electrónico tendría para dejar de fumar, a partir de los resultados de un ensayo clínico publicado en la revista científica New England Journal of Medicine ${ }^{1}$, como miembros del Programa Nacional de Control de Tabaco (PNCT), perteneciente al Ministerio de Salud y Desarrollo Social de la Nación, consideramos necesario expresar algunas consideraciones para la comunidad médica y público general.
\end{abstract}

Teniendo en cuenta que este trabajo es un importante aporte de evidencia consideramos, sin embargo, que presenta limitaciones que menguan su capacidad para utilizarlo como herramienta en la toma de decisiones sobre recomendaciones terapéuticas de cesación tabáquica. Respecto a la validez interna, no incluye la posibilidad de que haya un doble ciego en la intervención, falta un reporte de la cantidad de nicotina administrada en la rama asignada a productos farmacológicos, existen posibles falsos negativos por el tipo de método de evaluación de la abstinencia, contaminación entre grupos, hay posibles diferencias en las expectativas de efecto entre los dos grupos que pueden haber influido sobre el resultado y la pérdida al seguimiento es de un $21 \%$ de los sujetos.

En relación a la validez externa, si bien esta publicación demuestra que los cigarrillos electrónicos tienen un mayor efecto que los parches de nicotina en lograr la cesación, se deben tener algunas precauciones al interpretar los resultados. Los participantes del estudio presentaban ciertas características particulares que podrían limitar la aplicabilidad del estudio a otros contextos: múltiples intentos previos de dejar, un consumo relativamente bajo de cigarrillos ( 15 unidades por día) y permanecieron usando nicotina en ambas ramas. Este uso persistente de nicotina es un hecho infrecuente en las clínicas de cesación, y plantea dificultades para la generalización de los resultados. Ambos grupos recibieron apoyo cognitivo conductual, lo cual no reproduce las circunstancias reales en que se utilizan los cigarrillos electrónicos, siendo muy común el uso recreacional y como puerta de entrada al consumo de tabaco y no como estrategia de cesación. Esta opinión concuerda con la de muchos referentes expertos en este tema ${ }^{2}$

En los resultados del estudio de referencia se encontró que el $80 \%$ de las personas que usaron cigarrillos electrónicos para dejar de fumar continuaron incorporando una dosis elevada de nicotina, cambiando el mecanismo de inhalación pero manteniendo la dependencia a esta sustancia adictiva ${ }^{3}$. Al menos un $33 \%$ de las personas que usaron cigarrillos electrónicos en este estudio reportaron usar tanques de nicotina con sustancias saborizantes, cuya inocuidad al ser inhalados aún no ha sido demostrada ${ }^{4}$.
La información procedente de otros estudios muestra que el efecto de los cigarrillos electrónicos en el mundo real es desalentar los intentos de dejar ${ }^{5,6}$. Existe evidencia que muestra que los usuarios de este tipo de dispositivos mantienen la adicción a la nicotina, dada la frecuencia del doble uso del cigarrillo electrónico más el cigarrillo convencional a lo largo del tiempo, con las implicancias que esto tiene para la salud". Esto limita claramente la evidencia de que sea un método eficaz para dejar de fumar. Además, tampoco puede afirmarse que los cigarrillos electrónicos sean inocuos ya que no existen aún suficiente cantidad de estudios independientes de la industria tabacalera que demuestren que sus consecuencias para la salud sean menores a largo plazo a las de un cigarrillo convencional ${ }^{8}$. Por el contrario, hay evidencia que los cigarrillos electrónicos emiten numerosas sustancias potencialmente tóxicas que exponen a sus usuarios a mayor riesgo cardiovascular ${ }^{9,10}$. Se ha demostrado que el aerosol (vapor) que emite el cigarrillo electrónico puede producir daño importante en el pulmón, incluyendo lesiones similares al enfisema (una de las enfermedades que causa enfermedad pulmonar obstructiva crónica o EPOC) $)^{11,12}$.

Bajo el discurso de "reducción de daño" sostenido por algunos profesionales de la salud, pero especialmente por la industria tabacalera, la venta de este tipo de dispositivo ha crecido exponencialmente en los últimos años ${ }^{13}$. Así, otra de las consecuencias graves del aumento en el uso de cigarrillos electrónicos a nivel mundial es que favorece el inicio del consumo en la población adolescente ${ }^{14,15}$. En 2017, el 11,7\% de los adolescentes de 13 a 17 años en los Estados Unidos, lo utilizaban. En este sentido, la Food and Drug Administration (FDA), la agencia regulatoria de ese país ha declarado como "epidémico" el uso de los cigarrillos electrónicos entre los jóvenes de su país. En Argentina, están en etapa de procesamiento los datos de la 5ta Encuesta de Tabaquismo en Jóvenes que se realizó a fines del 2018 que permitirán conocer la magnitud del uso de cigarrillos electrónicos en jóvenes en nuestro país. Un estudio realizado en unos 3000 adolescentes de CABA, Córdoba y Tucumán mostró que el 7,1\% había probado un cigarrillo electrónico ${ }^{18}$.

En la séptima reunión de la Conferencia de las Partes del Convenio Marco para el Control del Tabaco (COP7) de la Organización Mundial de la Salud realizada en el 2016 se invitó a los países a establecer distintas medidas para regular a los Sistemas Electrónicos de Administración de Nicotina (SEAN), como se denomina también a los cigarrillos electrónicos, entre las que se incluyen: la prohibición de importación, venta y distribución con los objetivos de prevenir la iniciación en el uso; minimizar el riesgo para la salud a usuarios y no usuarios; prevenir la declaración de beneficios no probados por los fabricantes y proteger a las actividades de control de tabaco ${ }^{12}$. Desde el año 2011 nuestro

\footnotetext{
* Integrante del Programa Nacional de Control del Tabaco. ajvidela@gmail.com

†Integrante del Programa Nacional de Control del Tabaco. msnbertoncini@gmail.com

‡ Integrante del Programa Nacional de Control del Tabaco. brunilda.ministerio@gmail.com

$\S$ Integrante del Programa Nacional de Control del Tabaco. paolamorello@ hotmail.com

**Integrante del Programa Nacional de Control del Tabaco. bettita79@gmail.com

$\dagger+$ Integrante del Programa Nacional de Control del Tabaco. nangospodinoff@gmail.com

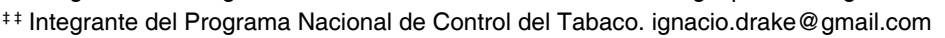


país cuenta con la disposición 3226/2011 de la Administración Nacional de Medicamentos, Alimentos y Tecnología Médica (ANMAT), que fuera ratificada en el 2016, y que, en su artículo 1ํ, dispone: "prohíbese la importación, distribución, comercialización y la publicidad o cualquier modalidad de promoción en todo el territorio nacional del sistema de administración de nicotina denominada "Cigarrillo Electrónico"...."

En función de estas consideraciones y de las limitaciones que presenta la publicación mencionada al principio, los miembros del PNCT consideramos que es precoz afirmar que los cigarrillos electrónicos son eficaces y seguros como estrategia para dejar de fumar. Sería importante contar con más información acerca del potencial que tienen los cigarrillos electrónicos de dañar la salud y de mantener la adicción a la nicotina antes de aceptarlos como un tratamiento para dejar de fumar. Recordamos que el sistema de salud cuenta con tratamientos para dejar de fumar cuya eficacia y seguridad ya ha sido demostrada por innumerables publicaciones científicas y que son aquellas que la Guía Nacional de Tratamiento de la Adicción al Tabaco recomienda ${ }^{16}$ Por eso, teniendo en cuenta el riesgo de daño de sus componentes, como la capacidad de iniciar en la adicción nicotínica a adolescentes y hasta contar con más información; se impone la aplicación del principio precautorio por el cual se deben tomar medidas que restrinjan actividades que puedan dañar la salud humana o el medio ambiente, aun si las relaciones causa/efecto no han sido totalmente establecidas científicamente ${ }^{17}$.

\section{Referencias}

1. Hajek P, y col. A Randomized Trial of E-Cigarettes versus Nicotine-Replacement Therapy. N Engl J Med [Internet]. 2019 Jan 30; Disponible en: https://doi.org/10.1056/NEJMoa1808779

2. Glantz SA. RCT comparing e-cigs to NRT shows effectiveness in a supervised smoking cessation program: what it does and does not mean. 2019.

3. Borrelli B, O'Connor GT. E-Cigarettes to Assist with Smoking Cessation. N Engl J Med [Internet]. 2019 Jan 30; Disponible en: https://doi.org/10.1056/NEJMe1816406

4. Tierney PA, y col. Flavour chemicals in electronic cigarette fluids. Tob Control [Internet]. 2016 Apr 1;25(e1):e10 LP-e15. Disponible en: http://tobaccocontrol.bmj.com/content/25/ e1/e10.abstract

5. Kalkhoran S, Glantz SA. E-cigarettes and smoking cessation in real-world and clinical settings: A systematic review and meta-analysis. Lancet Respir Med. 2016;4(2):116-28. 6. Hartmann-Boyce J, y col. Electronic cigarettes for smoking cessation. Cochrane Database Syst Rev [Internet]. 2016 Sep 13;(9). Disponible en: http://doi.wiley.com/10.1002/14651858. CD010216.pub3

7. Robertson L, y col. Dual use of electronic nicotine delivery systems (ENDS) and smoked tobacco: a qualitative analysis. Tob Control [Internet]. 2019 Jan 1;28(1):13 LP-19. Disponible en: http://tobaccocontrol.bmi.com/content/28/1/13.abstract

8. Agencia Nacional de Medicamentos Alimentos y Tecnologías Médicas. Seguridad y eficacia del cigarrillo electrónico en la práctica clínica. Informe ultrarrápido de evaluación de tecnología sanitaria. 2016

9. National Academies of Sciences and Medicine E. Public Health Consequences of E-Cigarettes [Internet]. Stratton K, Kwan LY, Eaton DL, editors. Washington, DC: The National Academies Press; 2018. Disponible en: https://www.nap.edu/catalog/24952/public-health-consequences-of-e-cigarettes

10. Alzahrani T, y col. Association Between Electronic Cigarette Use and Myocardial Infarction. Am J Prev Med [Internet]. 2018 Oct 1;55(4):455-61. Disponible en: https://doi. org/10.1016/j.amepre.2018.05.004

11. Ghosh A, y col. Chronic E-Cigarette Exposure Alters the Human Bronchial Epithelial Proteome. Am J Respir Crit Care Med [Internet]. 2018 Feb 26;198(1):67-76. Disponible en: https://doi.org/10.1164/rccm.201710-20330C

12. Garcia-Arcos I, y col. Chronic electronic cigarette exposure in mice induces features of COPD in a nicotine-dependent manner. Thorax [Internet]. 2016 Dec 1;71(12):1119 LP1129. Disponible en: http://thorax.bmj.com/content/71/12/1119.abstract

13. United States Department of Health and Human Services. E-Cigarette Use Among Youth and Young Adults: A Report of the Surgeon General. Department of Health and Human Services, Centers for Disease Control and Prevention, National Center for Chronic Disease Prevention and Health Promotion, Office on Smoking and Health. 2016.

Services, Centers for Disease Control and Prevention, National Center for Chronic Disease Prevention and Health Promotion, Office on Smoking and Health 2016. 14. Berry KM, y col. Association of Electronic Cigarette Use With Subsequent Initiation of Tobacco Cigarettes in US Youths. JAMA Netw Open [Internet]. 2019 Feb 1;2(2):e187794e187794. Disponible en: https://dx.doi.org/10.1001/jamanetworkopen.2018.7794

15. Cullen KA, y col. Tobacco Product Use Among Middle and High School Students — United States, 2011-2017. MMWR Morb Mortal Wkly Rep. 2018;67(22):629-33.

16. Ministerio de Salud y Desarrollo Social. Guía de Práctica Clínica de Tratamiento de la Adicción al Tabaco. Recomendaciones Basadas en la Evidencia Científica. 2011.

17. Grandjean P. Implications of the Precautionary Principle for Primary Prevention and Research. Annu Rev Public Health [Internet]. 2004 Mar 11;25(1):199-223. Disponible en: https://doi.org/10.1146/annurev.publhealth.25.050503.153941

18. Morello P, y col. Prevalence and predictors of e-cigarette trial among adolescents in Argentina. Tob. Prev. Cessation 2016;2(December):80 